\title{
COMBINATION OF N-GRAMS AND STOCHASTIC CONTEXT-FREE GRAMMARS FOR LANGUAGE MODELING*
}

\author{
José-Miguel Benedí and Joan-Andreu Sánchez \\ Departamento de Sistemas Informáticos y Computación \\ Universidad l'olitécnica de Valencia \\ Camino de Vora s/n, 46022 Valencia (Spain) \\ o-mail: \{jbenedi,jandreu\} (@dsic.upv.es
}

\begin{abstract}
This paper describes a hybrid proposial to combine n-grams and Stochastic Context-Free Grammars (SCFGs) for language modeling. A classical ne-gram model is used to captine the local relations between words, while a stochastic grammatical model is considered to represent the long-term relations between syntactical structures. In order to definc this grammatical model, which will be used on lange-vocabulary complex tasks, a category-based SCFG and a probabilistic model of word distribution in the categories have been proposed. Methods for learning these stochastic models for complex tasks are described, and algorithms for computing the word transition probabilitics are also presented. Finally, experiments using the Penn Treobank corpus improved by $30 \%$ the test set perplexity with regard to the cassical n-gram models.
\end{abstract}

\section{Introduction}

Language modeling is an important aspect to consider in large-vocabulary spech recognition systems (Bahl of al., 1983; Jelinck, 1998). The rl-gram models are the most widely-used for a wide range of domains (Bahl et al., 1983). The n-grams are simple and robust models and adequately capture the local restrictions between words. Moreover, it is well-known how to estimate the parameters of the model and how to integrate them in a speech recognition system. However, the 11-gram models cannot adequately characterizo the long-term constraints of the sentences of the tasks.

On the other hand, Stochastic Context-Free Grammars (SClGs) allow us a better model-

\footnotetext{
* This work has been partially supported by the Spanish CICYT under contract (TTC98/0423-C()6).
}

ing of long-term relations and work well on limited-domain tasks of low perplexity. Howcver, SCFGs work poorly for large-vocabulary, general-purpose tasks because learning $\mathrm{SCF}$ Gs and the computation of word transition probabilities present serious problems for complex real takks.

In the literature, a number of works have proposed ways to generalize the n-gram models (.Jelinck, 1998; Siu and Ostendorf, 2000) or combining with other structumal models (Bellegarda, 1998; Gilet and Ward, 1998; Cholba and Jelinek, 1998).

In this paper, we present a combined language model defined as a linear combination of n-grams, which are nsed to capture the local lelations between words, and a stochastic grammatical model which is used to represent the global relation between syntactic structures. In order to capture these long-term relations and to solve the main problems clerived from the largerocabulary complex taisks, we propose here to define: a catcgory-based SCFG and a probabilistic model of word distribution in the categories. Taking into account this proposal, we also describe here how to solve the learning of these stochastic models and their integration probloms.

With regard to the learning problem, several algorithms that learn SCFGs by means of ostimation algorithms lave been proposed (Lari and Young, 1990; Pereirat and Schabes, 1992; Sánchez and Benedí, 1998), and promising results have been achieved with category-based SCFGs on real tasks (Sánchez and Benedí, 1999).

In relation to the integration problem, we present two algorithms that compute the word trensition probability: the first algorithm is based on the Left-to-Right Inside algorithm 
(LRI) (Jclinek and Lafferty, 1991), and the second is based on an application of a Viterbi scheme to the LRI algorithm (the VLRI algorithm) (Sánchez and Benedí, 1997).

Finally, in order to evaluate the behavior of this proposal, experiments with a part of the Wall Street Journal processed in the Penn Treebank project were carried out and significant improvements with regard to the classical n-gram models were achieved.

\section{The language model}

An important problem related to language modeling is the evaluation of $\operatorname{Pr}\left(w_{k} \mid w_{1} \ldots w_{k-1}\right)$. In order to compute this probability, we propose a hybrid language model defined as a simple linear combination of n-gram models and a stochastic grammatical model $G_{s}$ :

$$
\begin{gathered}
\operatorname{Pr}\left(w_{k} \mid w_{1} \ldots w_{k-1}\right)=\alpha \operatorname{Pr}\left(w_{k} \mid w_{k-n} \ldots w_{k-1}\right) \\
+(1-\alpha) \operatorname{Pr}\left(w_{k} \mid w_{1} \ldots w_{k-1}, G_{s}\right),
\end{gathered}
$$

where $0 \leq \alpha \leq 1$ is a weight factor which depends on the task.

The expression $\operatorname{Pr}\left(w_{k} \mid w_{k-n} \ldots w_{k-1}\right)$ is the word probability of occurrence of $w_{k}$ given by the n-gram model. The parameters of this model can be easily estimated, and the cxpression $\operatorname{Pr}\left(w_{k} \mid w_{k-n} \ldots w_{k-1}\right)$ can be efficiently computed (Bahl et al., 1983; Jelinek, 1998).

In order to define the stochastic grammatical model $G_{s}$ of the expression $\operatorname{Pr}\left(w_{k} \mid w_{1} \ldots w_{k-1}, G_{s}\right)$ for large-vocabulary complex tasks, we propose a combination of two different stochastic models: a categorybased SCFG $\left(G_{c}\right)$, that allows us to represent the long-term relations between these syntactical structures and a probabilistic model of word distribution into categories $\left(C_{w}\right)$.

This proposal introduces two important aspects, which are the estimation of the parameters of the stochastic models, $G_{c}$ and $C_{w}$, and the computation of the following expression:

$$
\begin{aligned}
& \operatorname{Pr}\left(w_{k} \mid w_{1} \ldots w_{k-1}, G_{c}, C_{w}\right) \\
& \quad=\frac{\operatorname{Pr}\left(w_{1} \ldots w_{k} \ldots \mid G_{c}, C_{w}\right)}{\operatorname{Pr}\left(w_{1} \ldots w_{k-1} \ldots \mid G_{c}, C_{w}\right)}
\end{aligned}
$$

\section{Training of the models}

The parameters of the described model are estimated from a training sample, that is, from a set of sentences. Each word of the sentence has a part-of-speech tag (POStag) associated to it. These POStags are considered as word categories and are the terminal symbols of the SCFG. From this training sample, the parameters of $G_{c}$ and $C_{w}$ can be estimated as follows.

First, the parameters of $C_{w}$, represented by $\operatorname{Pr}(w \mid c)$, are computed as:

$$
\operatorname{Pr}(w \mid c)=\frac{\mathrm{N}(w, c)}{\sum_{w^{\prime}} \mathrm{N}\left(w^{\prime}, c\right)},
$$

where $\mathrm{N}(w, c)$ is the number of times that the word $w$ has been labcled with the POStag $c$. It is important to note that a word $w$ can belong to different categories. In addition, it may happen that a word in a test set does not appear in the training set, and therefore some smoothing technique has to be carried out.

With regard to the estimation of the categorybased SCFGs, one of the most widely-known methods is the Inside-Outside (IO) algorithm (Lari and Young, 1990). The application of this algorithm presents important problems which are accentuated in real tasks: the time complexity per iteration and the large number of iterations that are necessary to converge. An alternative to the IO algorithm is an algorithm based on the Viterbi score (VS algorithm) (Ncy, 1992). The convergence of the VS algorithm is faster than the IO algorithm. However, the SCFGs obtained are, in general, not as well learned (Sánchız ct al., 1996).

Another possibility for estimating SCFGs, which is somewhere between the IO and VS algorithms, has recently been proposed. This approach considers only a certain subset of derivations in the estimation process. In order to select this subset of derivations, two alternatives have been considered: from structural information content in a bracketed corpus (Pereira and Schabes, 1992; Amaya et al., 1999), and from statistical information content in the $k$ best derivations (Sánchez and Benedí, 1998). In the first alternative, the IOb and VSb algorithms which learn SCFGs from partially bracketed corpora were defined (Pereira and Schabes, 1992: Amaya et al., 1999). In the second alternative, the $k$ VS algorithm for the estimation of the probability distributions of a SCFG from the $k$-best derivations was proposed (Sánchez and Benedí, 1998). 
All of these algorithms have a time complexity $O\left(n^{3}\left|I^{\prime}\right|\right)$, where $n$ is the length of the input string, and $|P|$ is the size of the SCFG.

These algorithnns have been tested in real tasks for estimating category-based SCFGs (Sánche\% and Benedí, 1999) and the results obtained justify their application in complex real tasks.

\section{Integration of the model}

From expression (2), it can bee seen that in order to integrate the model, it is necessiry to efficiently compute the expression:

$$
\operatorname{Pr}\left(w_{1} \ldots w_{k} \ldots \mid G_{C}, C_{w}\right)
$$

In order to describe how this computation can be made, we first introduce some notation.

A Context-Free Grammar $G$ is a four-tuple $(N, \Sigma, P, S)$, where $N$ is the finite set of nonterminals, $\mathrm{Z}$ is the finite set of terminals $(N \cap \mathrm{\Sigma}=$ ()) $S \in N$ is the axion or initial symbol and $P$ is the finite set of productions or rules of the form $A \rightarrow \alpha$, where $A \in N$ and $\alpha \in(N \cup \Sigma)^{\dagger}$. (only grammars with non empty rules are considered). For simplicity (but without loss of generality) only context-free grammans in Chomshy Normal Form are considered, that is, grammin's with rules of the form $A \rightarrow B C$ or $A \rightarrow v$ where $A, B, C \in N$ and $v \in \Sigma$ :

A Stocthastic Context-Hite Grammat $G_{s}$ is a pair $(G, p)$, where $G$ is a context-free grammas and $p: P \rightarrow] 0,1]$ is a probability finc:tion of rule application such that $\forall A \in N$ : $\sum_{\alpha \in(N \cup \Sigma)+} p(A \rightarrow \alpha)=1$.

Now, we present two algorithms in order to compute the word transition probability. The first algorithm is based on the IJRI algorithm, and the second is based on an application of a Viterbi scheme to the LRI algorithm (the VLRI algorithm).

\section{Probability of generating an initial substring}

The computation of (4) is based on an algorithm which is a modification of the I,RI algorithm (Jelinck and Lafferty, 1991). This new algorithm is based on the definition of $\operatorname{Pr}(A<<$ $i, j))=\operatorname{Pr}\left(A \stackrel{*}{\Rightarrow} w_{i} \ldots w_{j} \ldots \mid G_{C}, C_{w}\right)$ as the probability that $A$ gencrates the initial substring $w_{i} \ldots u_{j} \ldots$ given $G_{c}$ and $C_{w}$. This can be conputed with the following dynannic programming schemo:

$$
\begin{gathered}
\operatorname{Pr}(A<<i, i)=\sum_{c}\left(p(A \rightarrow c) \operatorname{Pr}\left(w_{i} \mid c\right)\right. \\
\left.+\sum_{D} Q(A \Rightarrow D) p(D \rightarrow c) \operatorname{Pr}\left(w_{i} \mid c\right)\right) \\
\operatorname{Pr}(A<<i, j)=\sum_{l, C \in N} \sum_{l=i}^{j-1} Q(A \Rightarrow B C) \\
\operatorname{Pr}(B<i, l>) \operatorname{Pr}(C<<l+1, j) .
\end{gathered}
$$

In this way, $\operatorname{Pr}\left(w_{1} \ldots w_{k} \ldots \mid G_{c}, C_{w}\right)=$ $\operatorname{Pr}\left(S<<1, h_{i}\right)$.

In this expression, $Q(A \Rightarrow D)$ is the probability that $D$ is the leftmost nonterminal in all sentential forms which are derived from $A$. The value $Q(A \Rightarrow B C)$ is the probability that $B C$ is the initial substring of all sentential forms derived from $A \cdot \operatorname{l} r(B<i, l>)$ is the probability that the substring $w_{i} \ldots w_{l}$ is gencrated fiom $B$ given $G_{c}$ and $C_{u}$. Its computation will be defined later.

It should be noted that the combination of the models $G_{c}$ and $C_{w}$ is carried out in the value $\operatorname{Pr}(A<<i, i)$. This is the main difference with respect the J,RI algorithm.

\section{Probability of the best derivation gencrating an initial substring}

An algorithm which is similar to the previous one can be defined based on the Viterbi scheme. In this way, it is possible to obtain the best parsing of an initial substring. This new algorithm is also relatied to the VLRI algorithm (Sínchez and Benedí, 1997) and is based on the definition of $\widehat{\operatorname{Pr}}(A<<i, j))=\widehat{\operatorname{Pr}}\left(A \stackrel{*}{\Rightarrow} w_{i} \ldots w_{j} \ldots \mid G_{c}, C_{w}\right)$ as the probability of the most probable parsing which generates $w_{i} \ldots w_{j} \ldots$ from $A$ given $G_{c}$ : and $C_{w}$. This can be computed as follows:

$$
\begin{gathered}
\widehat{\operatorname{Pr}}(A<<i, i)=\max _{c}\left(p(A \rightarrow c) \operatorname{Pr}\left(w_{i} \mid c\right),\right. \\
\left.\max _{D}\left(\widehat{Q}(A \Rightarrow D) p(D \rightarrow c) \operatorname{Pr}\left(w_{i} \mid c\right)\right)\right), \\
\widehat{\operatorname{Pr}}(A<<i, j)=\operatorname{mix}_{l, C \in N} \operatorname{mix}_{l=i \ldots j-1}(\widehat{Q}(A \Rightarrow B C) \\
\widehat{\operatorname{Pr}}(B<i, l>) \widehat{\operatorname{Pr}}(C<<l+1, j)) .
\end{gathered}
$$

Therefore $\widehat{\operatorname{Pr}}\left(w_{1} \ldots w_{k} \ldots \mid G_{c}, C_{u}\right)=\widehat{\operatorname{Pr}}(S<<$ $1, k)$.

In this expression, $\widehat{Q}(A \Rightarrow D)$ is the probability that $D$ is the leftmost nonterminal in 
the most probable sentential form which is derived from $A$. The value $\widehat{Q}(A \Rightarrow B C)$ is the probability that $B C$ is the initial substring of most the probable sentential form derived from A. $\widehat{\operatorname{Pr}}(B<i, l>)$ is the probability of the most probable parse which gencrates $w_{i} \ldots w_{l}$ from $B$.

\section{Probability of generating a string}

The value $\operatorname{Pr}(A<i, j>)=\operatorname{Pr}(A \stackrel{*}{\Rightarrow}$ $\left.w_{i} \ldots w_{j} \mid G_{c}, C_{w}\right)$ is defined as the probability that the substring $w_{i} \ldots w_{j}$ is generated from $A$ given $G_{c}$ and $C_{w}$. To calculate this probability a modification of the woll-known Inside algorithm (Lari and Young, 1990) is proposed. This computation is carried out by using the following dynamic programming scheme:

$$
\begin{array}{r}
\operatorname{Pr}(A<i, i>)=\sum_{c} p(A \rightarrow c) \operatorname{Pr}\left(w_{i} \mid c\right), \\
\operatorname{Pr}(A<i, j>)=\sum_{B, C \in N} \sum_{l=i}^{j-1} p(A \rightarrow B C) \\
\operatorname{Pr}(B<i, l>) \operatorname{Pr}(C<l+1, j>) .
\end{array}
$$

In this way, $\operatorname{Pr}\left(w_{1} \ldots w_{n} \mid G_{c}, C_{w}\right)=\operatorname{Pr}(S<$ $1, n>$ ).

As we have commented above, the combination of the two parts of the grammatical model is carricd out in the value $\operatorname{Pr}(A<i, i>)$.

\section{Probability of the best derivation generating a string}

The probability of the best derivation that generates a string, $\widehat{\operatorname{Pr}}\left(w_{1} \ldots w_{n} \mid G_{c}, C_{w}\right)$, can be evaluated using a Viterbi-like scheme (Ney, 1992). As in the previous case, the computation of this probability is based on the definition of $\widehat{\operatorname{Pr}}(A<i, j>)=\widehat{\operatorname{Pr}}\left(A \stackrel{*}{\Rightarrow} w_{i} \ldots w_{j} \mid G_{c}, C_{w}\right)$ as the probability of the best derivation that gencrates the substring $w_{i} \ldots w_{j}$ from $A$ given $G_{c}$ and $C_{w}$. Similarly:

$$
\begin{aligned}
& \widehat{\operatorname{Pr}}(A<i, i>)=\max _{\boldsymbol{c}} p(A \rightarrow C) \operatorname{Pr}\left(w_{i} \mid c\right), \\
& \widehat{\operatorname{Pr}}(A<i, j>)=\max _{B, C \in N} \max _{l=i \ldots j-1} p(A \rightarrow B C) \\
& \widehat{\operatorname{Pr}}(B<i, l>) \widehat{\operatorname{Pr}}(C<l+1, j>) .
\end{aligned}
$$

Therefore, $\widehat{\operatorname{Pr}}\left(w_{1} \ldots w_{n} \mid G_{c}, C_{w}\right)=\widehat{\operatorname{Pr}}(S<$ $1, n>)$.

Finally, the time complexity of these algorithms is the same as the algorithms they are related to, therefore the time complexity is $O\left(k^{3}|P|\right)$, where $k$ is the length of the input string and $|P|$ is the size of the SCFG.

\section{Experiments with the Penn Treebank Corpus}

The corpus used in the experiments was the part of the Wall Strect Journal which had been processed in the Penn Treobank project ${ }^{1}$ (Marcus et al., 1993). This corpus consists of English texts collected from the Wall Strect Journal from editions of the late eighties. It contains approximately one million words. This corpus was automatically labelled, analyzed and manually checked as described in (Marcus et al., 1993). There are two kinds of labelling: a POStag labelling and a syntactic labelling. The size of the vocabulary is greater than 25,000 different words, the POStag vocabulary is composed of 45 labels $^{2}$ and the syntactic vocabulary is composed of 14 labcls.

The corpus was divided into sentences accord- ing to the bracketing. In this way, we obtained a corpus whose main characteristics are shown in Table 1.

Table 1: Characteristics of the Penn Treebank corpus once it was divided into sentences.

\begin{tabular}{|c|c|c|c|c|}
\hline $\begin{array}{c}\text { No. of } \\
\text { scnten. }\end{array}$ & $\begin{array}{c}\text { Av. } \\
\text { length }\end{array}$ & $\begin{array}{c}\text { Std. } \\
\text { deviation }\end{array}$ & $\begin{array}{c}\text { Min. } \\
\text { length }\end{array}$ & $\begin{array}{c}\text { Max. } \\
\text { length }\end{array}$ \\
\hline \hline 49,207 & 23.61 & 11.13 & 1 & 249 \\
\hline
\end{tabular}

We took advantage of the category-based SCFGs estimated in a previous work (Sáncher and Benedí, 1998). These SCFGs were estimated with sentences which had less than 15 words. Therefore, in this work, we assumed such restriction. The vocabulary size of the new corpus was 6,333 different words. For the experiments, the corpus was divided into a training corpus (directories 00 to 19) and a test corpus (directories 20 to 24). The characteristics of these sets can be seen in Table 2. The part of the

\footnotetext{
${ }^{1}$ Release 2 of this data set can be obtained from the Linguistic Data Consortium with Catalogue number LDC94T4B (http://www.ldc.upenn.edu/ldc/nofiame.html)

${ }^{2}$ There are 48 labels defined in (Marcus et al., 1993), however, three of them do not appear in the corpus.
} 
corpus labeled with POStags was used to ostimate the parameters of the grammatical mockel, while the non-labeled part was used to estimate the parameters of tho n-gram model. Wo now describe the estimation process in detail.

Table 2: Characteristics of the data sets defined for the experiments whon the sentences with 1urore than 15 POStags wore removert.

\begin{tabular}{|c|c|c|c|}
\hline $\begin{array}{c}\text { Dalia } \\
\text { set }\end{array}$ & $\begin{array}{c}\text { No. of } \\
\text { senten. }\end{array}$ & $\begin{array}{c}\text { Av. } \\
\text { length }\end{array}$ & $\begin{array}{c}\text { Std. } \\
\text { deviation }\end{array}$ \\
\hline \hline Training & 9.933 & 10.67 & 3.46 \\
\hline Test & 2,295 & 10.51 & 3.55 \\
\hline
\end{tabular}

The paranneters of a 3-grann model were ostimated with the software tool describer in (Roscufeld, 1995) ${ }^{3}$. We used tho linear interpolation smooth teclnicque supported by this tool. The ont-of-vocabulary words wore gromed in the same cletss and were nsed in tho compntation of the perplexity. 'lles test set perjolexity with this model wats 180.4 .

Tlue values of expression (3) wore computex from the tanged and non-tatgged part of the training corpus. In order to avoid null values, the unseon events were laboled with a special symbol $w^{\prime}$ which dicl not appear in the vocabulary, in such a way that $\operatorname{Pr}\left(u^{\prime} \mid c\right) \neq 0$, $\forall c \in C$, where $C$, was the sed of calogonies. That is, all the categorios conld gonemate tilo nu. socm event. 'This probability took a very small value (sovelal orders of magnitude less than

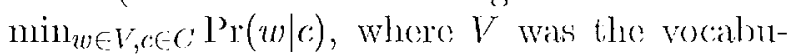
lasy of the training corpus), and differont values of this probability did not change the results.

The paranoters of an initial ergodic SCFG were estimated with each one of the estimation methods mentioned in Section 3. 'This SCFG had 3,374 rules, composed from 45 terminal symbols (the number of l'OStags) and 14 nonterminal symbols (the number of syntactic latbels). The probabilitios wore randomly generated and thee different seeds were tested, but only one of them is reported given that the results were very similar. 'The training corpus was the labeled part of the described corpus. The perplexity of the labeled part of the test set for

\footnotetext{
${ }^{3}$ Release 2.04 is available at htte//svrwww.eng.cam.ac.ukk/ pre14/toolkit.htınl.
}

different estimation algorithms an be seen in Thalle 3.

Table 3: Perplexity of the labeled part of the test set with the SC]C estimated with the mothods montioned in Section 3.

\begin{tabular}{|c|c|c|c|}
\hline VS & $k \mathrm{VS}$ & IOb & VSb \\
\hline \hline 21.56 & 20.65 & 13.14 & 21.84 \\
\hline
\end{tabular}

Once we had estimated the paranneters of the dofined model, we applied expression (1) by using the liRl algorithm and tho VLRI algorithm in expression (4). 'The test set perplexity that: was obtained in function of $\alpha$ for different estimation algorithms (VS, $k$ VS, IOb and VSb) can be seen in lig. 1.

In the best case, the proposed language model oblained more than a $30 \%$ improvenent orer results obtained by the 3-gram langnage model (soe Jable 4). 'This result was obtianed when the SCHG estimated with the IOb algorithm was resed. 'Ihe SCICGs estimated with ottier algorithms also obtained innortant imporovenontis compared to the 3-gram. In adelition, it cam be observed that both the I.RI algorithm and the VISI algorithm obtained good results.

Table 4: Best tost perplexity for different $\mathrm{SCFG}$ estination algorithns, and the perentage of inprovenent with respect to the 3 -grane nuodel.

\begin{tabular}{|c|c|c|c|c|}
\hline & VS & liVS & $\mathrm{IO} \mathrm{b}_{3}$ & $\bar{V} \overline{S b}$ \\
\hline ISRI & $133 . \overline{6}$ & 130.3 & 124.6 & 136.3 \\
\hline$\%$ improv. & $25.9 \%$ & $27.8 \%$ & $30.9 \%$ & $24.5 \%$ \\
\hline VIRI & 143.4 & 137.2 & 132.4 & 149.7 \\
\hline$\%$ improv. & $20.5 \%$ & $23.0 \%$ & $26.6 \%$ & $17.0 \%$ \\
\hline
\end{tabular}

An importinit aspect to note is that the weight of the grammatical part was approximately $50 \%$, which mcans that this part provicled important information to the language model.

\section{Conclusions}

A new langniage model has been introduced. This new language model is defined as a linear combination of an n-gram which representis relations between words, and a stochastic 

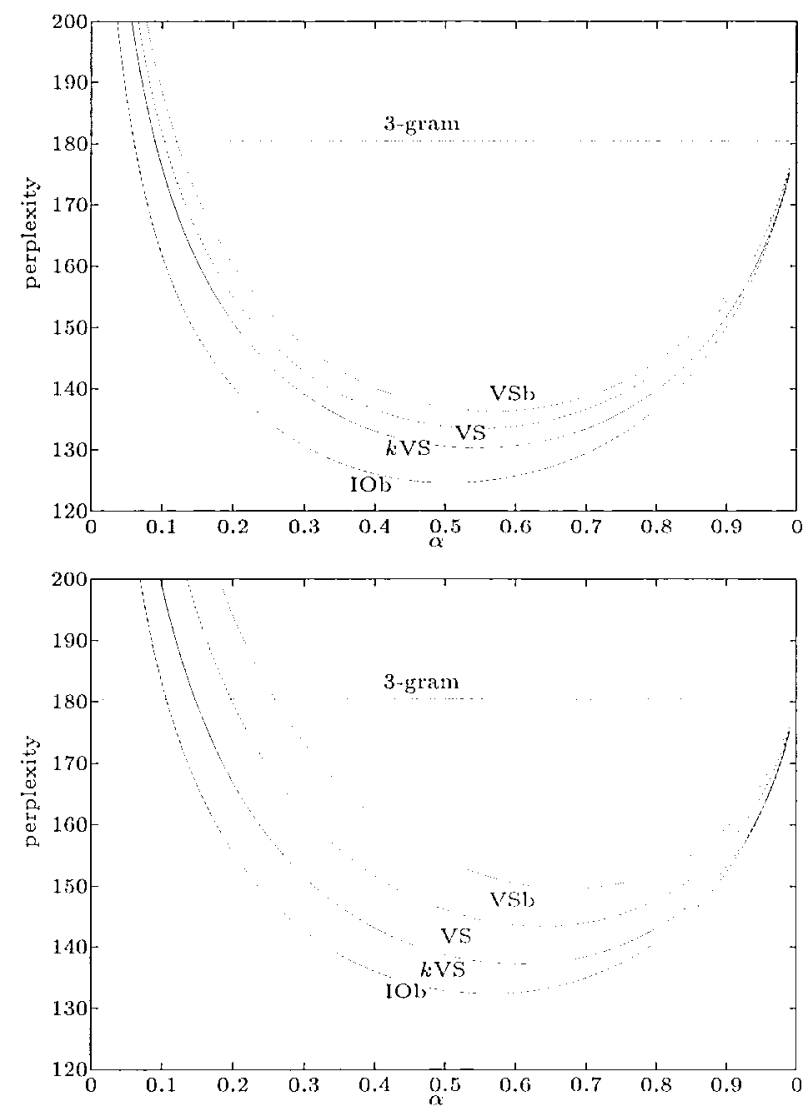

Figure 1: Test set perplexity obtained with the proposed language models in function of gamma. Different curves comespond to SCFGs estimated with different algorithms. The upper graphic corresponds to the results obtained when the LRI algorithm was used in the language models, and the lower graphic corresponds to the results obtained with the VLRI algorithm.

grammatical model which is used to represent the global relation between syntactic structures. The stochastic grammatical model is composed of a category-based SCFG and a probabilistic model of word distribution in the categories. Several algorithms have been described to estimate the parameters of the model from a the sample. In addition, efficient algorithms for solving the problem of the interpretation with this model have been presented.

The proposed model has been tested on the part of Wall Street Journal processed in the Penn Treebank project, and the results obtained improved by more than $30 \%$ the test set per- plexity over results obtained by a simple 3-gram model.

\section{References}

F. Amaya, J.M. Bencdí, and J.A. Sánchez. 1999. Learning of stochastic context-frec grammars from brackoted corpora by means of reestimation algorithms. In M.I. Torres and A. Sanfoliu, editors, Proc. VIII Spanish Symposium on Pattern Recognition and Image Analysis, pages 119-126, Bilbao, España, May. AERFAI.

L.R. Bahl, F. Jelinck, and R.I. Mercer. 1983. A maximum likelihood approach to continuous speech recognition. IEEE Trans. Pattern Analysis and Machine Intelligence, PAMI5(2):179-190.

J.R. Bellegarda. 1998. A multispan language modeling framework for large vocabulary speech recognition. IEEE Trans. Speech and Audio Processing, 6(5):456-476.

C. Chelba and F. Jelinck. 1998. Exploiting syntactic structure for language modeling. In Proc. COLING, Montreal, Canada. University of Montreal.

J. Gilet and W. Ward. 1998. A language model combining trigrams and stochastic contextfree grammars. In In 5th International Conference on Spoken Language Processing, pages 2319-2322, Sidney, Australia.

F. Jelinek and J.D. Lafferty. 1991. Computation of the probability of initial substring generation by stochastic context-free grammars. Computational Linguistics, 17(3):315-323.

F. Jelinek. 1998. Statistical Methods for Speech Recognition. MIT Press.

K. Lari and S.J. Young. 1990. The estimation of stochastic context-free grammars using the inside-outside algorithm. Computer, Speech and Language, 4:35-56.

M.P. Marcus, B. Santorini, and M.A. Marcinkiewicz. 1993. Building a large annotated corpus of english: the penn treebank. Computational Linguistics, 19(2):313-330.

H. Ney. 1992. Stochastic grammars and pattorn recognition. In P. Laface and R. De Mori, editors, Speech Recognition and Understanding. Recent Advances, pages 319-344. SpringerVerlag.

F. Pcrcira and Y. Schabes. 1992. Insideoutside reestimation from partially brack- 
eted corpora. In Procedings of the $30 t h$ Annual Mecting of the Association for Computational Linguistics, pages 128-135. University of Delaware.

R. Rosenfeld. 1995. The cmun statistical language modeling toolkit and its use in the 1994 arpa csr cvaluation. In ARPA Spoken Language Technology Workshop, Austin, Texas, USA.

M. Siu and M. Ostendorf. 2000. Variable n-grams and extensions for conversational speech language modding. IEEE Trans. on Speceh and Audio Processing, 8(1):63 75 .

J.A. Sánche\% and J.M. Benedí. 1997. Computation of the probability of the best derivation of an initial substring from a stochastic contextfree grammar. In A. Sanfelin, J..J. Villanueva, and J. Vitria, editors, Proc. VII Spanish Symposium on Pattern Recognition and Image Analysis, pages 181 186, Barcelona, España, April. AERFAI.

J.A. Sanche\% and J.M. Benedí. 1998. Estimation of the probability distributions of stochastic context-free granmars from the $k$ best derivations. In In 5th International Conference on Spoken Language Processing, pages 24952498 , Sidney, Australia.

J.A. Sánchoz and J.M. Benedí. 1999. Learning of stochastic context-free grammars by means of estimation algorithms. In P'roce. EUROSPELCH'99, volume 4, pages 1799 1802, Budapest, Hungary.

J.A. Sánchoz, J.M. Bonedí, and F. Casacuberta. 1996. Comparison botween the insideoutside algorithm and the viterbi algorithm for stochastic context-fiec grammars. In P. Perner, P. Wang, and A. Rosenfold, editors, Advances in Structural and Syntactical Pattern Recognition, pages 50 59. Sp) ringerVorlag. 